\title{
Transfer-Free In Situ CCVD Grown Nanocrystalline Graphene for Sub-PPMV Ammonia Detection
}

\author{
Dennis Noll, ${ }^{1, z}$ Philipp Hönicke, ${ }^{2}$ Yves Kayser, ${ }^{2}$ Stefan Wagner, ${ }^{3}$ Burkhard Beckhoff, ${ }^{2}$ \\ and Udo Schwalke ${ }^{1, *}$ \\ ${ }^{1}$ Institute for Semiconductor Technology and Nanoelectronics, Technische Universität Darmstadt, 64289 Darmstadt, \\ Germany \\ ${ }^{2}$ Physikalisch-Technische Bundesanstalt (PTB), 10589 Berlin, Germany \\ ${ }^{3}$ Chair of Electronic Devices, RWTH Aachen University, 52074 Aachen, Germany
}

Reasonably-priced devices for the detection of toxic species in the atmosphere are critical for reasons of health. Previous research work shows the promising detection capabilities of graphene. Thus, we demonstrate the gaseous response of our nanocrystalline graphene field-effect transistors that can be fabricated hundredfold on a two inch substrate by our transfer-free in situ catalytic chemical vapor deposition process. By means of Raman spectroscopy and near edge X-ray absorption fine structure nanocrystallinity of the CCVD grown graphene films within the devices can be confirmed. Using a self-constructed vacuum probing station the sensitivity of the fabricated devices is extracted from dynamic electrical sampling measurements. With respect to ammonia it is found that the sensitivity is being higher than previously reported from other groups. Moreover, a comparable responsivity is achieved. A deeper understanding of the origin of the high sensitivity, which we attribute to the nanocrystallinity, is given by backgate input characteristics under varying ammonia concentration as well as from comparison with literature results on carbon nanotube gas sensors. Furthermore, the origin and influence of ammonia on the hysteresis of our nanocrystalline graphene field-effect transistors is discussed.

(C) The Author(s) 2018. Published by ECS. This is an open access article distributed under the terms of the Creative Commons Attribution Non-Commercial No Derivatives 4.0 License (CC BY-NC-ND, http://creativecommons.org/licenses/by-nc-nd/4.0/), which permits non-commercial reuse, distribution, and reproduction in any medium, provided the original work is not changed in any way and is properly cited. For permission for commercial reuse, please email: oa@ electrochem.org. [DOI: 10.1149/2.0171807jss]

(cc) BY-NC-ND

Manuscript submitted February 14, 2018; revised manuscript received April 5, 2018. Published April 13, 2018. This paper is part of the JSS Focus Issue on Semiconductor-Based Sensors for Application to Vapors, Chemicals, Biological Species, and Medical Diagnosis.

For reasons of health protection the demand for environmental monitoring systems for the surveillance of harmful toxic gases and vapors in the ambient atmosphere has increased over the recent years. For such applications solid-state gas sensors are favored as they can show significant changes in their electronic properties upon contact with a toxin. Furthermore, they provide a highly scalable approach with low production costs. ${ }^{1,2}$ In addition, appropriate solid-state gas sensors provide the possibility of monolithic integration within silicon circuitry thus allowing the fabrication of compact gas monitoring systems at low cost. In order to provide such sensors with as high as possible sensitivities, semiconducting nanomaterials with high surface-to-volume ratios are preferred since the ratio of the surface conductivity in relation to the bulk material conductivity is highest. ${ }^{3}$ In this case, a change of the surface Fermi energy and thus surface conductivity through charge transfer of absorbed gaseous species, ${ }^{4,5}$ leads to a maximized change of the total conductivity.

Graphene, a two-dimensional carbon nanomaterial with one of the highest surface-to-volume ratios, has first been experimentally demonstrated by K. Novoselov and A. Geim in the year $2004 .{ }^{6}$ Due to its hexagonal $\mathrm{sp}^{2}$ hybridized honeycomb lattice structure graphene shows a linear dispersion relationship with a "zero bandgap" at the K points of the Brillouin zone. ${ }^{6-8}$ Accordingly, small changes in the Fermi energy can already lead to significant changes in the charge carrier densities and consequently the electronic properties. For example, by measuring the hall resistivity of a graphene device fabricated through the micromechanical cleavage of graphite, the excellent sensitivity has been demonstrated by the detection of single molecules of nitrogen dioxide $\left(\mathrm{NO}_{2}\right){ }^{9}$ Additionally, other research groups have published sensors on the basis of chemical vapor deposition (CVD) graphene grown on copper or nickel substrates that has been transferred to oxidized silicon substrates, ${ }^{10-13}$ reduced graphene oxide, ${ }^{14-16}$ or epitaxi-

\footnotetext{
*Electrochemical Society Member
}

${ }^{\text {z} E-m a i l: ~ n o l l @ i h t . t u-d a r m s t a d t . d e ~}$ ally grown graphene on silicon carbide (SiC) wafers. ${ }^{17,18}$ Nevertheless, the origin of the mechanism, modifying the conductivity in graphene, is not completely understood. So far, studies have shown that the gaseous sensitivity of graphene devices seems to have their origin in residual surface adsorbates, ${ }^{19}$ grain boundaries and defects in the graphene crystal structure ${ }^{11,12,20}$ as well as defects in the underlying substrate. ${ }^{21}$ In this context we have investigated the gaseous sensitivity of our nanocrystalline graphene field-effect transistors (ncGFETs), which are fabricated by means of a PMMA-enhanced transfer-free in situ catalytic chemical vapor deposition (CCVD) process. ${ }^{22,23} \mathrm{By}$ this method the ncGFETs are directly grown in their final positions and can be electrically contacted through their respective lithographically patterned metal catalyst sites (see Fig. 1c). Consequently, processing or cleaning steps after the graphene deposition process can be avoided.

\section{Device Fabrication}

Fabrication of our ncGFETs is done using a $2^{\prime \prime}$ silicon complementary metal-oxide-semiconductor (CMOS) process line, complemented with the CCVD process for the graphene deposition. In the first place, a silicon dioxide backgate dielectric is fabricated by dry thermal oxidation, followed by the spin deposition of an additional carbon source consisting of a $20 \mathrm{~nm}$ thin polymethyl methacrylate (PMMA) layer. Subsequently, a lift-off photoresist is lithographically patterned prior to the electron beam evaporation of the metal catalyst composed of thin films of aluminum and nickel, each with a thickness of $10 \mathrm{~nm}$ (see Fig. 1a). Meanwhile, the PMMA in direct contact with the metal becomes annealed. By the use of an ultrasonic assisted heated solvent bath the lift-off photoresist and non-annealed PMMA is removed, leaving behind the dedicated PMMA-metal catalyst sites (see Fig. 1b). The substrates are then annealed in nitrogen gas at a temperature of $900^{\circ} \mathrm{C}$ to initiate metal grain growth and cluster formation. ${ }^{22}$ Additionally, the PMMA is pyrolyzed whereby the carbon becomes dissolved in the metal catalyst. ${ }^{24}$ After 5 minutes of annealing methane $\left(\mathrm{CH}_{4}\right)$ and 


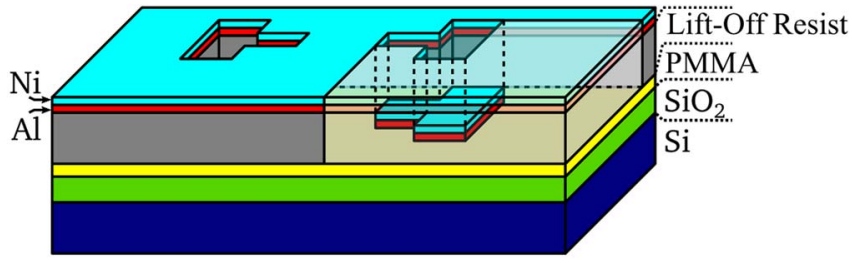

a
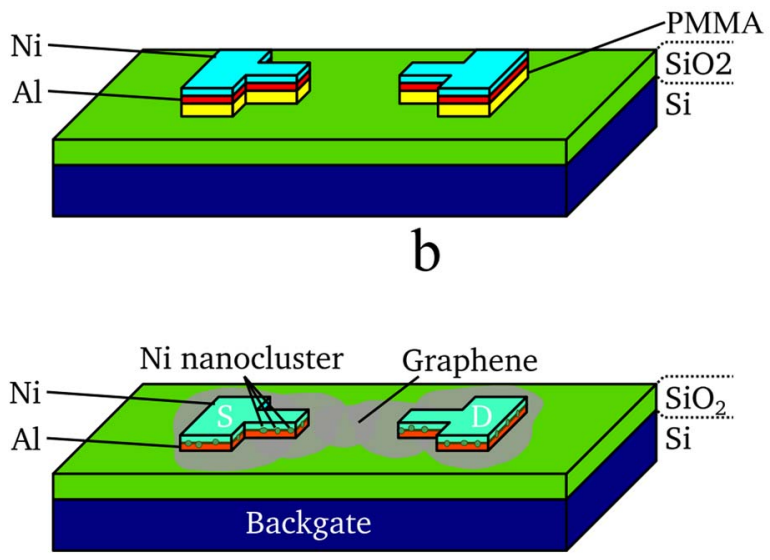

C

Figure 1. Schematic flow for the fabrication of transfer-free in situ CCVD nanocrystalline graphene field-effect transistors. (a) Patterned lift-off photoresist after evaporation of aluminum and nickel catalyst thin films (b) PMMAmetal catalyst structures after Lift-Off (c) Final schematic representation of ncGFET with respective source, drain and backgate terminals.

hydrogen $\left(\mathrm{H}_{2}\right)$ are added into the CVD reactor for 10 minutes. During this time the nanocrystalline graphene grows laterally extending over the borders of the metal catalysts connecting the dedicated catalyst sites. The final substrates are then again cooled down in nitrogen gas yielding the final ncGFETs that are ready for use (see Fig. 1c). Figure 2 shows the respective photography and micrograph of an oxidized two inch silicon substrate, containing hundreds of functional ncGFETs, suitable for gas detection.

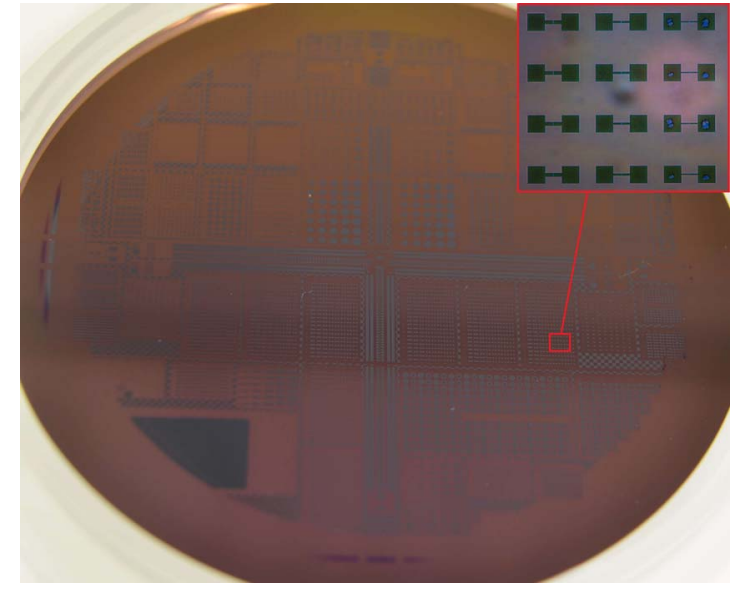

Figure 2. Oxidized two inch silicon substrate containing hundreds of functional ncGFETs. The bluish areas show the respective catalyst sites used as electrical contacts as can be seen in the micrograph inset.

\section{Materials Characterization}

The analysis of the in situ CCVD grown material was done using a Horiba Labram HR 800 Raman spectrometer equipped with a $632.8 \mathrm{~nm}$ red laser. Figure 3 shows typical Raman spectra that have been acquired between the source and drain electrode terminals of different ncGFETs. By comparison of our Raman spectra with those from Ferrari et al. ${ }^{25}$ we can deduce on the $\mathrm{G}$ peak resonance at around $1600 \mathrm{~cm}^{-1}$ and the defect induced D peak at $1350 \mathrm{~cm}^{-1}$. However the measured $\mathrm{G}$ peak position of our nanocrystalline graphene is higher than that of a pristine monolayer of graphene, which has been determined to a wavenumber of around $1580 \mathrm{~cm}^{-1}$ by Ferrari et al. ${ }^{25}$ According to Schmidt et al., who have analyzed nanocrystalline graphene, this up-shift can be explained as follows: ${ }^{26}$ By the existence of chain defects in the graphitic structure a secondary defect induced resonance peak at around $1620 \mathrm{~cm}^{-1}$ appears that is superimposed with the primary $\mathrm{G}$ peak resonance. ${ }^{26}$ As a consequence of the high density of defects the $2 \mathrm{D}$ peak at $2630 \mathrm{~cm}^{-1}$ is highly damped and split into the additional D + D' resonance at $2910 \mathrm{~cm}^{-1} \cdot{ }^{25,26} \mathrm{In}$ addition, $532 \mathrm{~nm}$ Raman mapping of the $\mathrm{G}\left(1605 \mathrm{~cm}^{-1}\right)$ and 2D peak $\left(2700 \mathrm{~cm}^{-1}\right)$ around the source and drain catalyst sites has been performed. Visually enhanced Raman maps are shown in Figs. 4a and 4b,

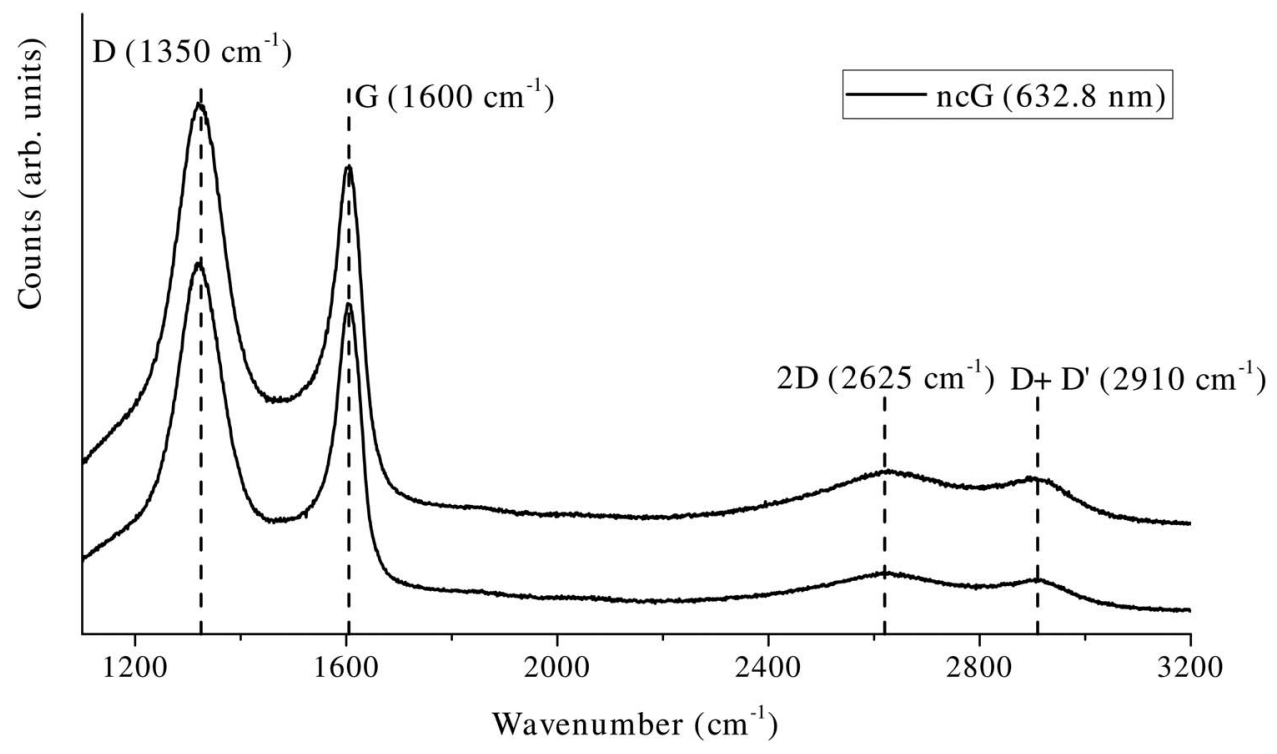

Figure 3. Raman spectra $(632.8 \mathrm{~nm})$ of typical ncGFETs measured between their respective source and drain terminals. 

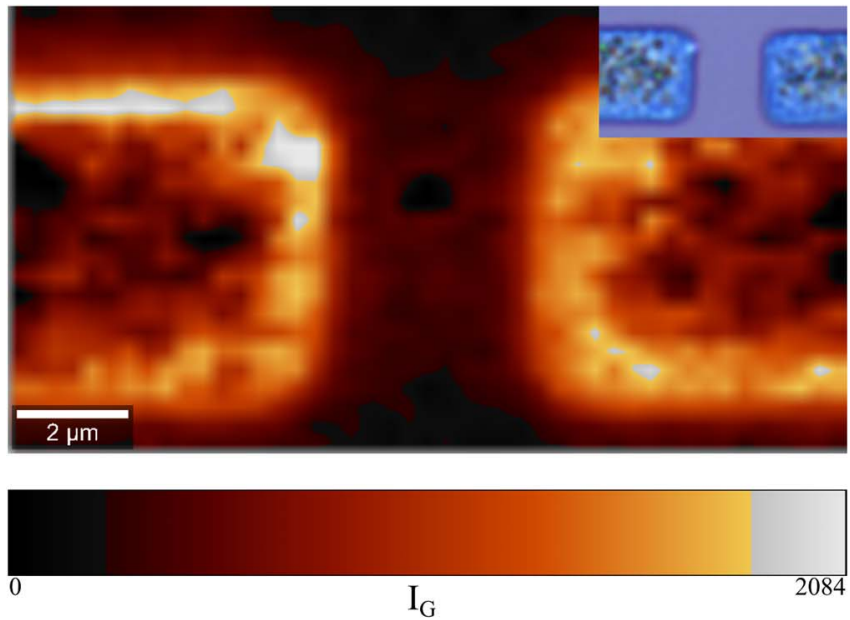

(a)
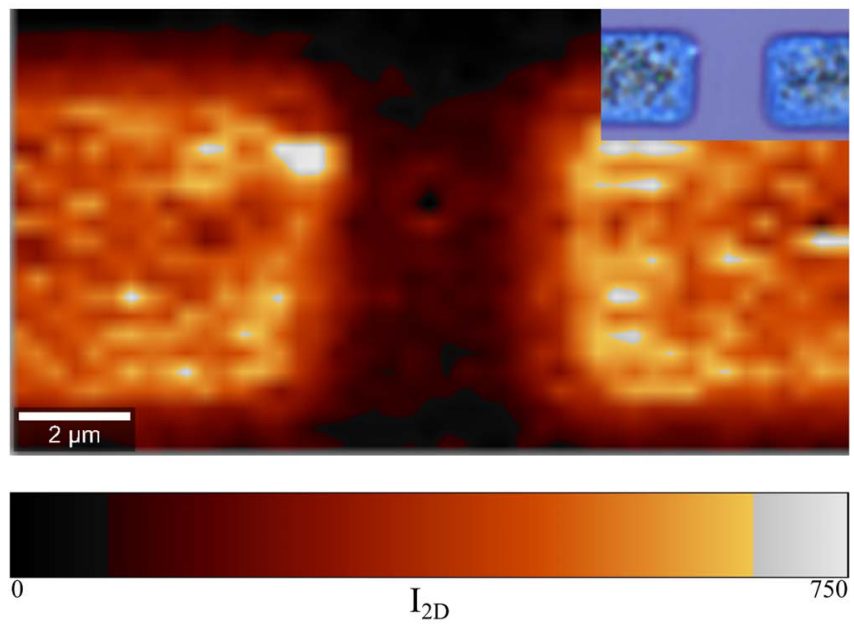

(b)

Figure 4. Raman maps $(532 \mathrm{~nm})$ measured between source and drain catalyst sites of a ncGFET (a) Intensity of $\mathrm{G}$ peak at $1605 \mathrm{~cm}^{-1}$ (b) Intensity of 2D peak at $2700 \mathrm{~cm}^{-1}$.

confirming a preferential growth of nanocrystalline graphene between the source and drain regions of the devices.

In order to further discriminate between nanocrystalline graphene and graphite the thickness of the nanocrystalline CCVD graphene film has been measured by atomic force microscopy (AFM). For this purpose, a step has been created by contact-mode scratch removal of the nanocrystalline graphene that has been measured afterwards using tapping-mode AFM. The resulting step profile is shown in Fig. 5 ensuring a thickness of approximately $1 \mathrm{~nm}$ consistent with 3 layers of graphene.

Furthermore, the $\mathrm{C}-\mathrm{C} \mathrm{sp} \mathrm{sp}^{2}$ carbon structure of our nanocrystalline CCVD graphene was investigated by means of near edge X-ray absorption fine structure (NEXAFS) analysis at the carbon K-edge. For comparison purposes reference samples of graphene transferred onto $300 \mathrm{~nm} \mathrm{SiO} 2 / \mathrm{Si}$ and a water dispersion of $0.5 \mathrm{mg} / \mathrm{ml}$ graphene oxide have been purchased from Graphenea. For the measurements a graphene oxide film has been spin deposited onto a $\mathrm{SiO}_{2} / \mathrm{Si}$ wafer from the dispersion. The NEXAFS experiments were performed at the plane grating monochromator $(\mathrm{PGM})^{27}$ beamline of PTB at the BESSY II synchrotron employing radiometrically calibrated instrumentation. ${ }^{28,29}$ Figure 6 shows the fluorescence yield spectra of the NEXAFS analysis that have been normalized with respect to the incident photon flux in the photon energy regime between 297 and $300 \mathrm{eV}$. For all three samples one can observe the $\pi^{*}$ resonance being

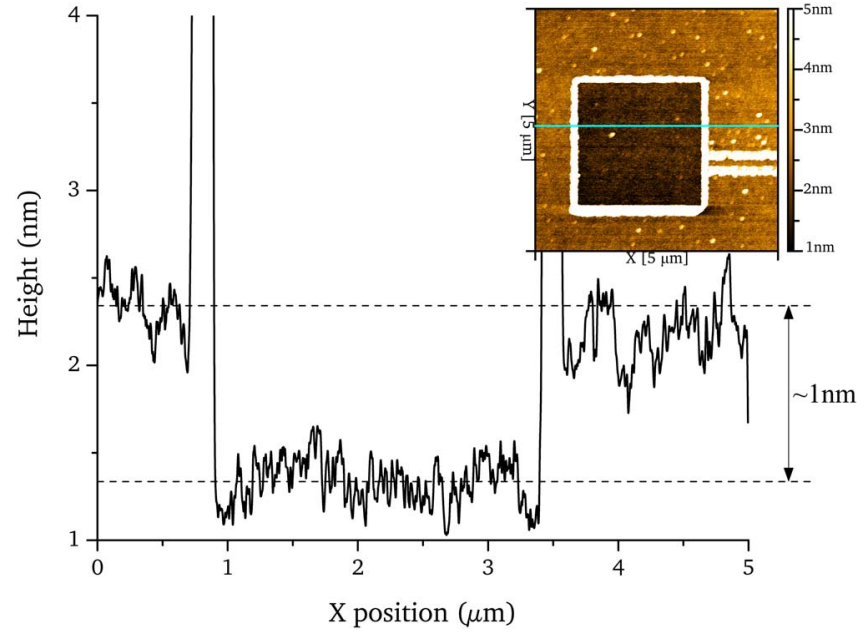

Figure 5. Profile line measurement of AFM thickness of nanocrystalline graphene near catalyst.

consistent with the flat molecular orientation of the graphene sheets on their respective surfaces.$^{30}$ Considering the $\pi^{*} / \sigma^{*}$ ratio, which is an indication for the $\mathrm{sp}^{2}$ carbon fraction, ${ }^{31}$ the highest ratio can be found for the reference graphene sample with a value of $\pi^{*} / \sigma^{*}=1.69$, followed by our nanocrystalline graphene with a ratio of $\pi^{*} / \sigma^{*}=1.54$. The lowest ratio of $\pi^{*} / \sigma^{*}=1.3$ is found for the reference graphene oxide sample, thus having a higher $\mathrm{sp}^{3}$ content due to functional oxygen groups. Furthermore, the reference graphene oxide sample shows a signature at around $286 \mathrm{eV}$, which is absent in the NEXAFS spectra of the reference graphene as well as the nanocrystalline graphene. When taking a look at the smaller peaks of each of the NEXAFS spectra we can identify a peak (A) at around $288.6 \mathrm{eV}$. From comparison with the work of Preobrajenski et al. ${ }^{32}$ we assign the origin of this peak to be caused from transition metals that may remain from the high temperature CVD growth process with catalysts made from either copper or nickel. ${ }^{33}$ Further evidence for this assumption is obtained from the NEXAFS spectra of nickel carbide, which may in our case be formed during the cool-down phase of our fabrication process. ${ }^{31,34}$ Another peak, which is solely found in the case of our nanocrystalline

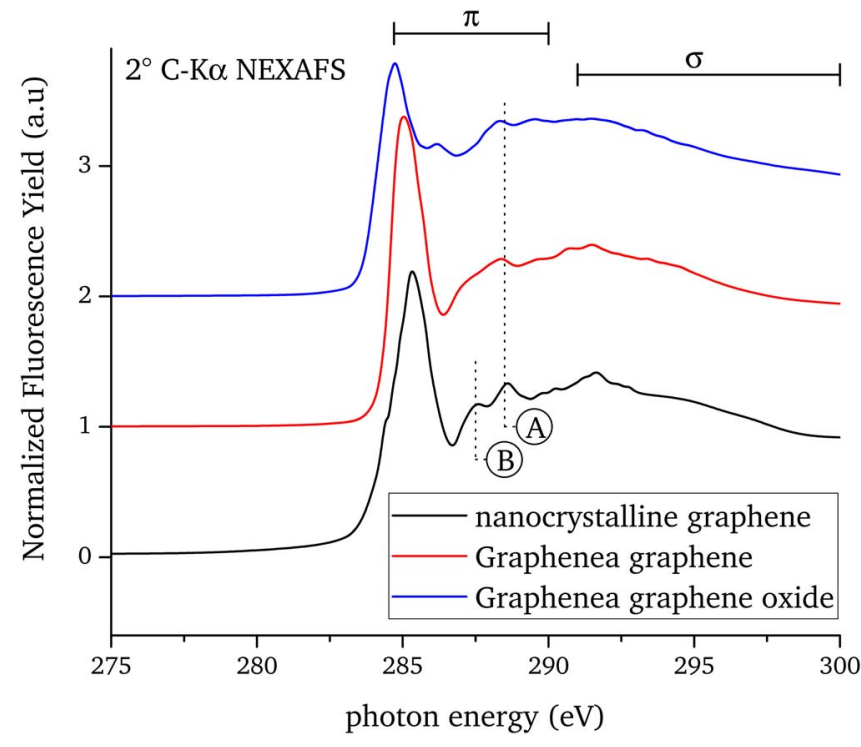

Figure 6. Near edge X-ray absorption fine structure spectra of CCVD nanocrystalline graphene, reference graphene on $\mathrm{SiO}_{2} / \mathrm{Si}$ and reference graphene oxide on $\mathrm{SiO}_{2} / \mathrm{Si}$ both supplied by Graphenea. 


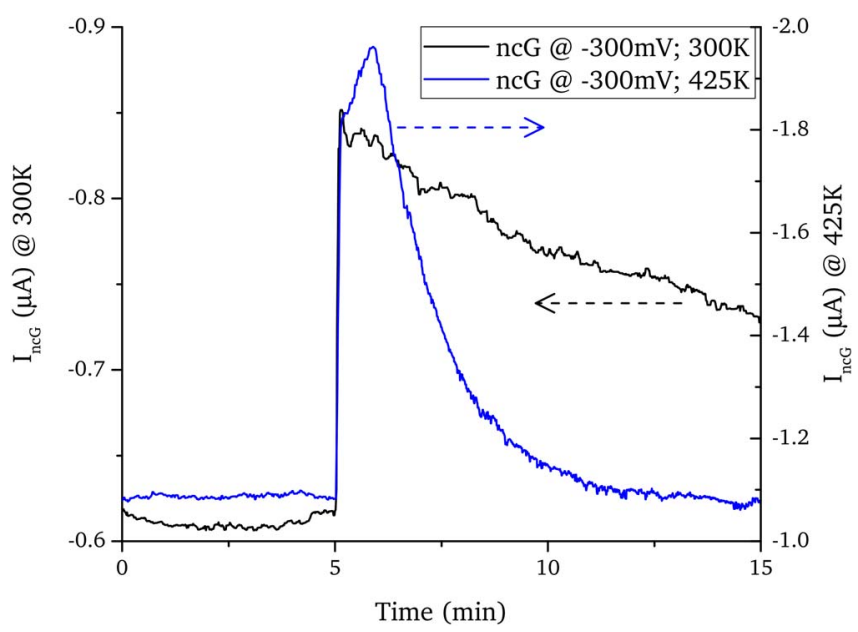

Figure 7. Dynamic response of a ncGFET upon exposure to 4 ppmv ammonia in vacuum at $300 \mathrm{~K}$ room temperature (black line, left scale) and $425 \mathrm{~K}$ (blue line, right scale).

graphene, is positioned at around $287.5 \mathrm{eV}$ (B). Under consideration of our fabrication process using nitrogen gas we suggest that this peak is related to incorporated nitrogen as evident from the work of Iyer et al. as well as Geng et al. ${ }^{35,36}$

To briefly summarize the presented results: (I) The thickness of the CCVD grown material is $1 \mathrm{~nm}$ or less. (II) The G and comparatively high $\mathrm{D}$ peak intensity of the Raman spectra indicate a high density of defects in the CCVD grown carbon film thus leading to a highly damped and split 2D peak. (III) The Raman maps of $\mathrm{G}$ and 2D peaks confirm the preferential growth between the source and drain catalyst sites. (IV) Spectra of NEXAFS measurements of our CCVD grown films are very similar to reference spectra obtained from graphene samples provided from Graphenea, both with strong $\pi^{*}$ and weak $\sigma^{*}$ resonances confirming a flat molecular orientation, which is made from C-C sp ${ }^{2}$ carbon. ${ }^{30}$ Accordingly, we are confident that our CCVD grown material is nanocrystalline graphene, similar to that used in the work of Sun et al. ${ }^{37}$ and other research groups. ${ }^{26,38-41}$ Furthermore, transmission electron microscopy (TEM) images and selected area electron diffraction patterns (SAED) from a focused ion beam cut have been previously investigated and published. ${ }^{42}$ From these measurements the growth of layered graphene with a basal plane distance of $\sim 3.5 \AA$ has been confirmed. Finally, additional evidence is obtained from conductive AFM measurements which have revealed the presence of numerous electrical defects in the topologically smooth conductive nanocrystalline graphene film. ${ }^{43}$

\section{Ammonia Detection}

For the characterization of the sensitivity of our ncGFETs toward ammonia a self constructed half-automatic wafer probing station inside a vacuum chamber is used. Inside the vacuum chamber a base pressure of $p=4 \mathrm{E}-5$ mbar is achieved, which can be systemically flooded with calibrated gas mixtures through a mass flow controller (MFC). The volume concentration of the gaseous species inside of the vacuum chamber can then be derived from the vacuum pressure by using the ideal gas law. For the electrical characterization the source measurement units of an HP4156A semiconductor parameter analyzer are connected with the respective metallic source and drain catalyst sites of the ncGFETs as well as the wafer chuck for the global backgate contact.

The dynamic response of a typical grounded backgate $\mathrm{V}_{\mathrm{G}}=0 \mathrm{~V}$ ncGFET at a drain-to-source bias of $\mathrm{V}_{\mathrm{DS}}=-300 \mathrm{mV}$ is shown in Fig. 7. The demonstrated typical ncGFET sensor exhibits a nominal device width of $\mathrm{W}=100 \mu \mathrm{m}$ and a device length of $\mathrm{L}=3 \mu \mathrm{m}$. After 5 minutes of sampling of the intrinsic current flow the device

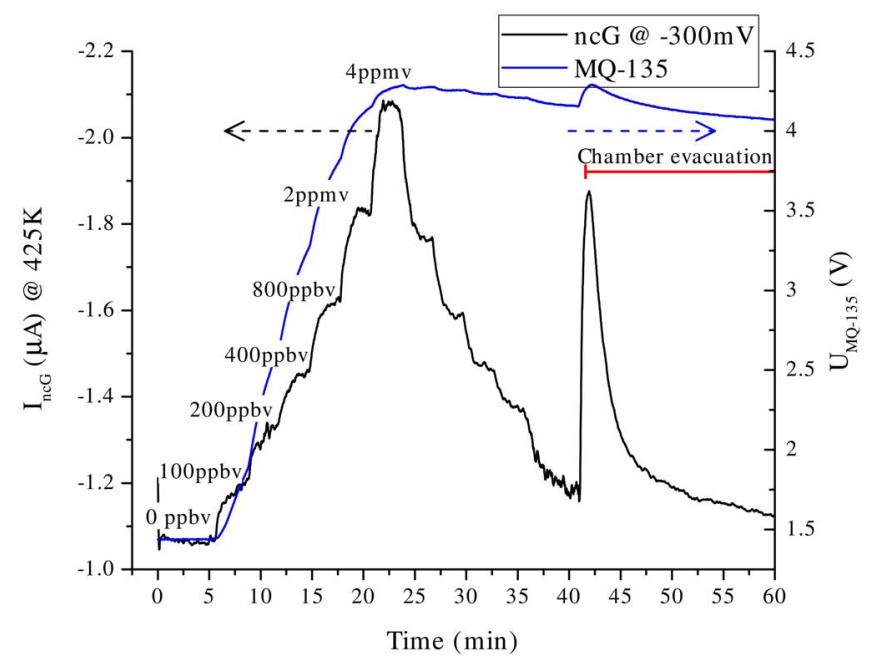

Figure 8. Sensitivity of a ncGFET upon exposure to different volume concentration of ammonia at elevated temperature of $425 \mathrm{~K}$ (black line, left scale) in comparison with $\mathrm{SnO}_{2}$-based MQ-135 reference sensor (blue line, right scale).

has been exposed to a single pulse of 4 parts-per-million-volume (ppmv) ammonia (Air Liquide $\mathrm{NH}_{3}$, purity $99.999 \%$ ) at $300 \mathrm{~K}$ room temperature (black line, left scale) and $425 \mathrm{~K}$ (blue line, right scale). Subjected to this ammonia pulse the device shows a steep increase of the electrical conductivity leading to a room temperature sensitivity of $\mathrm{S}_{4 \mathrm{ppmv}, 300 \mathrm{~K}}=39.5 \%$, where the sensitivity is defined as the ratio of the change in conductivity over the intrinsic electrical conductivity, as described by Equation 1 .

$$
\mathrm{S}=\left(\mathrm{G}-\mathrm{G}_{0}\right) / \mathrm{G}_{0}
$$

In comparison with other research groups, who reported sensitivities of e.g. $\mathrm{S}_{1 \mathrm{ppm}} \approx 4 \%{ }^{9}$ or $\mathrm{S}_{10 \mathrm{ppm}} \approx 15 \%,{ }^{12}$ our sensitivity is rather high, comparable with that of a graphene oxide functionalized $\mathrm{ZnO}$ sensor. ${ }^{16}$ We attribute this increased sensitivity of our devices to the nanocrystallinity and thus high density of grain boundaries in our graphene. This assignment is confirmed by Yasaei et al., ${ }^{11}$ who analyzed the sensitivity of individual graphene grain boundaries. Nevertheless, only a very slow recovery of the initial state is observed at room temperature. However, at elevated temperatures recovery is enhanced as was seen by Schedin et al., ${ }^{9}$ who annealed their graphene device at $150^{\circ} \mathrm{C}$ for the recovery. For that reason we have repeated the single pulse exposure of our ncGFET at a temperature of $425 \mathrm{~K}$. In this case the ncGFET shows the same steep response, achieving an even higher sensitivity of $\mathrm{S}_{4 \mathrm{ppmv}, 425 \mathrm{~K}}=80.6 \%$. Furthermore, the ncGFET shows a considerably faster recovery, reaching the intrinsic current flow after 7.5 minutes. Please note, that in this case the recovery speed is limited by the evacuation rate of our vacuum chamber.

Subsequently, we have determined the detection limits as well as the linearity of our ncGFET ammonia sensors at a temperature of $425 \mathrm{~K}$ by varying the volume concentrations of $\mathrm{NH}_{3}$, as depicted in Fig. 8 (black line, left scale). We observed that the ncGFETs are capable of achieving stable levels of conductivity after 3 minutes of exposure for each volume concentration tested, regardless of the direction of pressure change, i.e. increasing or decreasing $\mathrm{NH}_{3}$ concentration. However, the sensor response is not linear with respect to $\mathrm{NH}_{3}$ volume concentration and conductivity change. As limited by calibration gas leakage through the MFC, the minimum concentration level which could be clearly detected is 100 parts-per-billion-volume (ppbv). Moreover, from the electrical sampling measurement of Fig. 8 , the transient behavior in terms of the rise time $t_{90}$ and the fall time $t_{10}$ is investigated. The response times are defined as the durations until $90 \%$ of the upper or $110 \%$ of the lower current thresholds are achieved, respectively. By this method a rise time of $t_{90} \approx 60$ 


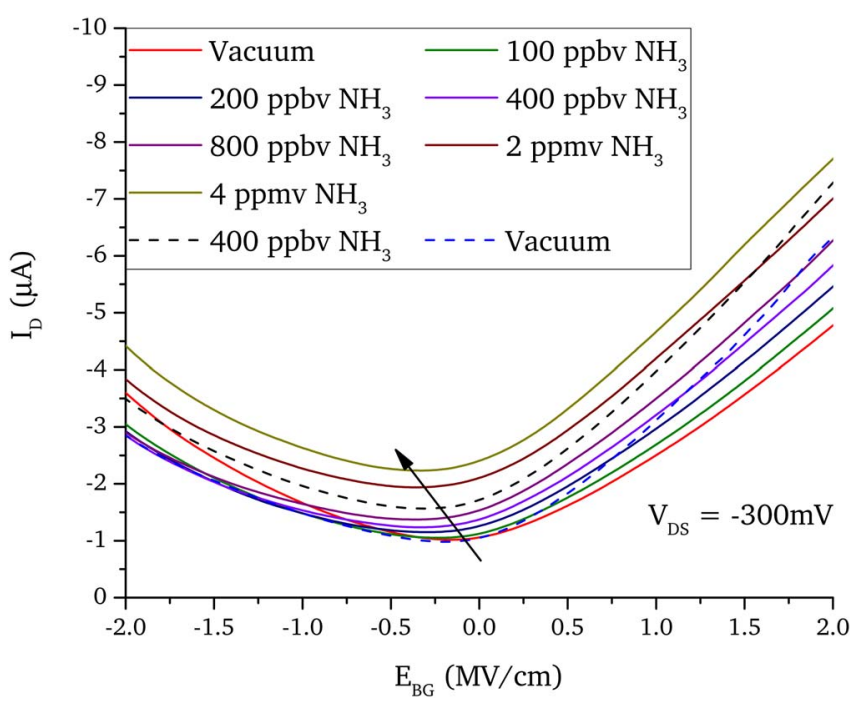

Figure 9. Backgate input characteristics of a ncGFET at $425 \mathrm{~K}$ for the different volume concentrations of ammonia used for the sensitivity measurement. The solid lines lines indicate the branch of increasing ammonia concentration whereas the dashed lines show the decreasing branch.

$\mathrm{s}$ can be extracted for any of the ammonia concentration changes, whereas the fall time shows a variation of $t_{10} \approx 65 \pm 10 \mathrm{~s}$. For comparison purposes the voltage signal of a commercial $\mathrm{SnO}_{2}$-based MQ-135 ammonia sensor has been recorded as can also be seen in Fig. 8 (blue line, right scale). The MQ-135 does not attain stable voltage signal levels for the various volume concentrations of ammonia. Note that the reference sensor does not recover due to the absence of oxygen. Anyway, the specifications of the MQ-135 do not cover vacuum applications and requires at least $2 \%$ oxygen for calibrated operation.

To obtain a deeper understanding of the mechanism of the gaseous species on the electrical conductivity of our ncGFETs, we have measured backgate input characteristics as shown in Fig. 9. Each input characteristic has been recorded after 3 minutes of exposure to their respective volume concentration, following the measurement procedure of the previous sampling measurement. For clarity we have omitted the measurement results of 2 ppmv, 800 ppbv, 200 ppbv, and 100 ppbv of the branch of decreasing ammonia concentrations in Fig. 9. Therefore, a time of 9 minutes has passed between the measurement of the $4 \mathrm{ppmv}$ and dashed 400 ppbv input characteristics. The final, dashed input characteristic of the ncGFET in vacuum has been taken after a total time of 60 minutes, cf. dashed lines in Fig. 9.

All input characteristics of our ncGFET show ambipolar behavior, as is typical for graphene devices. . $9,12,38$ This means the majority of the current is induced by means of electron transport on the right branch or hole transport on the left branch respectively. The charge neutrality point is the position of minimum conductivity and described by the position at which the dominating transport regime changes from holes to electrons or vice versa. Considering the ammonia exposure we can see that the charge neutrality point becomes shifted toward a more negative backgate potential indicating increased electron doping. This is in accordance with previous results from other research groups. ${ }^{9,12}$ However, we also observe a global increase of the current flow implying a secondary mechanism besides charge transfer doping. An analogous phenomenon of a global conductivity increase was seen by Boyd et al., who analyzed the $\mathrm{NO}_{2}$ sensitivity of carbon nanotube (CNT) networks with varying amounts of junctions among the CNT. In their case the rise in conductivity is explained by the lowering of the potential barrier that is located at the junction between two $\mathrm{CNT}^{44} \mathrm{~A}$ similar effect is also known from the grain boundary potential barriers of polycrystalline $\mathrm{SnO}_{2}$-based sensors. ${ }^{45}$ As the junctions among linked CNT can be considered similar to grain boundaries we suggest that the exposition toward ammonia modifies the grain boundary potential barriers ${ }^{46}$

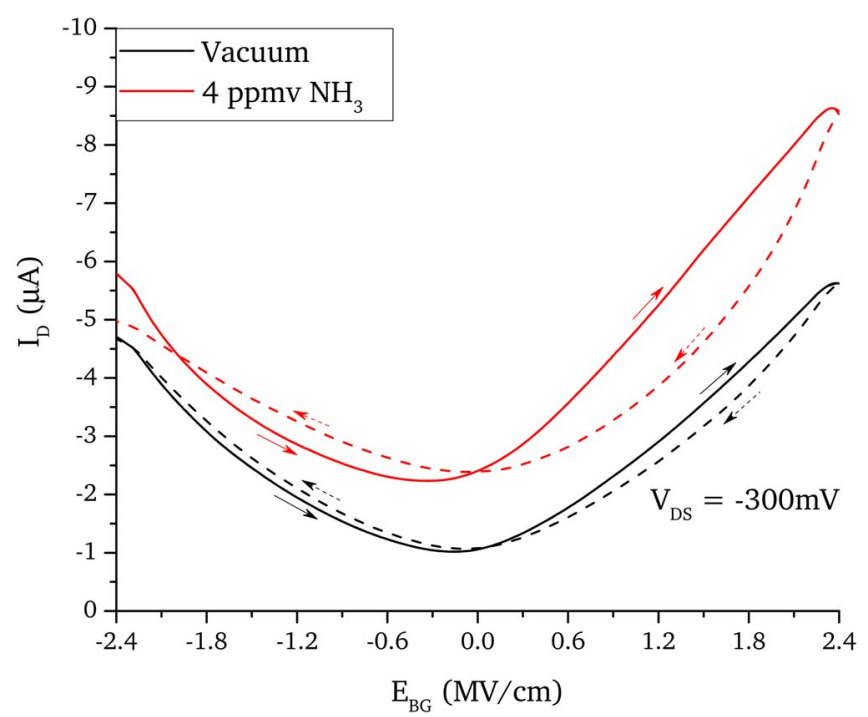

Figure 10. Hysteresis in the backgate input characteristics of a ncGFET at $425 \mathrm{~K}$ in vacuum and under exposure to 4 ppmv of ammonia.

of our nanocrystalline graphene, thus leading to the increased current flow and higher sensitivity of our ncGFETs. Additionally, we also observe a change of the slope of the current with respect to the backgate electric field, thus implying changes of the effective charge carrier field-effect mobilities. These changes may also be issued to modifications of the grain boundary potential barriers, which will affect the current transport in the nanocrystalline graphene increasing the error in the extraction of the charge carrier field-effect mobility. In view of these restrictions, a low value of the effective charge carrier mobility in the range of $1 \mathrm{~cm}^{2} / \mathrm{Vs}$ has been extracted, which is typical for nanocrystalline graphene. ${ }^{38}$ However, since the ncGFETs are intended for gas sensor applications, the primary focus is not on the mobility rather than the high surface-to-volume ratio for high detection sensitivity. Reconsidering the recovery of our devices we can see that the conductivity for the grounded backgate ncGFET has returned to its initial value. Furthermore, the charge neutrality point is reverted to its intrinsic value thus reducing the electron doping effect of the ammonia on the nanocrystalline graphene. Nevertheless, the current slope in respect to the backgate electric field has not recovered, which we account to strongly bound adsorbents at the grain boundaries. This of course may influence the dynamic response of our ncGFETs in the case of being actively driven with a backgate bias to increase the gaseous sensitivity.

Furthermore, the hysteresis of our ncGFET has been monitored ranging from $-2.4 \mathrm{MV} / \mathrm{cm}$ to $2.4 \mathrm{MV} / \mathrm{cm}$ and reverse as is indicated by the arrows in Fig. 10. Despite the fact of the electrical characterization within a vacuum probing station we still observe an intrinsic hysteresis loop with a positively shifted charge neutrality point. Upon exposure with ammonia the hysteresis loop becomes larger and a higher charge neutrality point shift is observed between the forward and reverse backgate bias sweeps. Two contrasting mechanisms have been proposed by Wang et al. for the origin of the hysteresis. ${ }^{47}$ For one thing charge trapping at the graphene/ $\mathrm{SiO}_{2}$ interface, and for another thing capacitive gating from the charging of dipoles within the $\mathrm{SiO}_{2} \cdot{ }^{47}$ Another mechanism discussed by Joshi et al. is the formation of a gate series capacitance in the upper region of the $\mathrm{SiO}_{2}$ that is based on charge traps with nonequivalent capture and emission rates. ${ }^{48}$ Since we only observe a positive shift of the charge neutrality point capacitive gating can mostly be neglected. ${ }^{47}$ Nevertheless, the surface adsorbed ammonia possesses a high dipole moment that might be influential next to its electron doping effect on the nanocrystalline graphene layer. However, a more thorough investigation on the transient response of the hysteresis is still necessary. 


\section{Conclusions}

Hundreds of nanocrystalline graphene FETs applicable to gas detection have been fabricated on a two inch silicon substrate by transferfree in situ catalytic CVD. By means of Raman spectroscopy, conductive atomic force microscopy ${ }^{43}$ and near edge X-ray absorption fine structure analysis the material properties of our CCVD graphene have been studied. By means of these characterization methods, an approximately $1 \mathrm{~nm}$ thin, defect rich carbon layer with high C-C $\mathrm{sp}^{2}$ carbon content has been identified. The obtained NEXAFS spectrum is very similar to that of a pristine graphene on the $\mathrm{SiO}_{2} / \mathrm{Si}$ reference supplied by Graphenea. In addition, previously published TEM images and SAED results show the formation of layered carbon with a basal plane distance of approx. $3.5 \AA$, as observed for stacked graphene within bulk graphite. ${ }^{42}$ Thus we consider the CCVD grown material to be made from nanocrystalline graphene. Nonetheless, the ambipolar field-effect is still observable for backgate controlled devices made from nanocrystalline graphene. The high sensitivity of our nanocrystalline graphene sensors toward ammonia in the ppbv to ppmv range is demonstrated by means of ncGFETs. From backgate input characteristics a global increase of the device conductivity is found in addition to the previously reported shift of the charge neutrality point. By comparison with earlier carbon nanotube research a similar effect is known from grain boundary potential modifications, which we attribute to be the source of the global conductivity increase. Furthermore, an enlargement of the hysteresis loop has been observed upon ammonia exposure that still needs further investigation in terms of its transient response.

\section{Acknowledgment}

The authors thank Prof. Dr.-Ing. Max Christan Lemme, Chair of Electronic Devices, from the RWTH Aachen University for the opportunity of recording Raman maps. Furthermore we thank PD Dr. Emanuel Ionescu and Benjamin Juretzka from Technische Universität Darmstadt for the opportunity to record single Raman spectra at the group of dispersive solids.

\section{ORCID}

Dennis Noll (D) https://orcid.org/0000-0002-3669-2657

\section{References}

1. R. Moos, K. Sahner, M. Fleischer, U. Guth, N. Barsan, and U. Weimar, Sensor (Basel), 9, 323 (2009)

2. X. Liu, S. Cheng, H. Liu, S. Hu, D. Zhang, and H. Ning, Sensors (Basel), 12, 9635 (2012).

3. W. Göpel, Prog Surf Sci, 20, 9 (1985).

4. W. Fu, L. Feng, G. Panaitov, D. Kireev, D. Mayer, A. Offenhausser, and H. J. Krause, Sci Adv, 3, e1701247 (2017).

5. G. S. Kulkarni, K. Reddy, Z. Zhong, and X. Fan, Nat Commun, 5, 4376 (2014).

6. K. S. Novoselov, A. K. Geim, S. V. Morozov, D. Jiang, Y. Zhang, S. V. Dubonos, I. V. Grigorieva, and A. A. Firsov, Science, 306, 666 (2004)

7. M. S. Dresselhaus and G. Dresselhaus, Advances in Physics, 51, 1 (2002).

8. T. Ando, Journal of the Physical Society of Japan, 74, 777 (2005)

9. F. Schedin, A. K. Geim, S. V. Morozov, E. W. Hill, P. Blake, M. I. Katsnelson, and K. S. Novoselov, Nat Mater, 6, 652 (2007).

10. I. F. Rivera, R. K. Joshi, and J. Wang, in 2010 IEEE Sensors, p. 1534 (2010).

11. P. Yasaei, B. Kumar, R. Hantehzadeh, M. Kayyalha, A. Baskin, N. Repnin, C. Wang, R. F. Klie, Y. P. Chen, P. Kral, and A. Salehi-Khojin, Nat Commun, 5, 4911 (2014).
12. S. M. Mortazavi Zanjani, M. M. Sadeghi, M. Holt, S. F. Chowdhury, L. Tao, and D. Akinwande, Applied Physics Letters, 108 (2016).

13. A. D. Smith, K. Elgammal, X. Fan, M. C. Lemme, A. Delin, M. Råsander, L. Bergqvist, S. Schröder, A. C. Fischer, F. Niklaus, and M. Östling, RSC Advances, 7, 22329 (2017)

14. G. Lu, L. E. Ocola, and J. Chen, Applied Physics Letters, 94 (2009).

15. V. Dua, S. P. Surwade, S. Ammu, S. R. Agnihotra, S. Jain, K. E. Roberts, S. Park, R. S. Ruoff, and S. K. Manohar, Angew Chem Int Ed Engl, 49, 2154 (2010).

16. N. A. A. A, R. K. Kampara, R. P. K, and J. B. G, Sensors and Actuators B: Chemical, 255, 1064 (2018).

17. P. K. Ang, W. Chen, A. T. S. Wee, and K. P. Loh, Journal of the American Chemical Society, 130, $14392(2008)$.

18. R. Pearce, T. Iakimov, M. Andersson, L. Hultman, A. L. Spetz, and R. Yakimova, Sensors and Actuators B: Chemical, 155, 451 (2011).

19. Y. Dan, Y. Lu, N. J. Kybert, Z. Luo, and A. T. Johnson, Nano Lett, 9, 1472 (2009).

20. A. Salehi-Khojin, D. Estrada, K. Y. Lin, M. H. Bae, F. Xiong, E. Pop, and R. I. Masel, Adv Mater, 24, 53 (2012).

21. B. Kumar, K. Min, M. Bashirzadeh, A. B. Farimani, M. H. Bae, D. Estrada, Y. D. Kim, P. Yasaei, Y. D. Park, E. Pop, N. R. Aluru, and A. Salehi-Khojin, Nano Lett, 13, 1962 (2013).

22. P. J. Wessely, F. Wessely, E. Birinci, U. Schwalke, and B. Riedinger, Journal of Vacuum Science \& Technology B, Nanotechnology and Microelectronics: Materials, Processing, Measurement, and Phenomena, 30 (2012).

23. D. Noll and U. Schwalke, ECS Transactions, 75, 11 (2016).

24. J. Kwak, T. Y. Kwon, J. H. Chu, J. K. Choi, M. S. Lee, S. Y. Kim, H. J. Shin, K. Park, J. U. Park, and S. Y. Kwon, Phys Chem Chem Phys, 15, 10446 (2013).

25. A. C. Ferrari, Solid State Communications, 143, 47 (2007).

26. M. E. Schmidt, C. Xu, M. Cooke, H. Mizuta, and H. M. H. Chong, Materials Research Express, 1, 025031 (2014).

27. F. Senf, U. Flechsig, F. Eggenstein, W. Gudat, R. Klein, H. Rabus, and G. Ulm, J Synchrotron Radiat, 5, 780 (1998)

28. B. Beckhoff, Journal of Analytical Atomic Spectrometry, 23, 845 (2008).

29. J. Lubeck, B. Beckhoff, R. Fliegauf, I. Holfelder, P. Hönicke, M. Müller, B. Pollakowski, F. Reinhardt, and J. Weser, Rev Sci Instrum, 84, 045106 (2013).

30. C. Mainka, P. S. Bagus, A. Schertel, T. Strunskus, M. Grunze, and C. Wöll, Surf Sci, 341, L1055 (1995).

31. A. Furlan, J. Lu, L. Hultman, U. Jansson, and M. Magnuson, J Phys Condens Matter, 26, 415501 (2014)

32. A. B. Preobrajenski, M. L. Ng, A. S. Vinogradov, and N. Mårtensson, Physical Review $B, 78$ (2008)

33. G. Lupina, J. Kitzmann, I. Costina, M. Lukosius, C. Wenger, A. Wolff, S. Vaziri, M. Östling, I. Pasternak, A. Krajewska, W. Strupinski, S. Kataria, A. Gahoi, M. C. Lemme, G. Ruhl, G. Zoth, O. Luxenhofer, and W. Mehr, ACS Nano, 9, 4776 (2015).

34. B. Pollakowski, P. Hoffmann, M. Kosinova, O. Baake, V. Trunova, R. Unterumsberger, W. Ensinger, and B. Beckhoff, Anal Chem, 85, 193 (2013).

35. G. R. Iyer, J. Wang, G. Wells, M. P. Bradley, and F. Borondics, Nanoscale, 7, 2289 (2015).

36. D. Geng, S. Yang, Y. Zhang, J. Yang, J. Liu, R. Li, T.-K. Sham, X. Sun, S. Ye, and S. Knights, Applied Surface Science, 257, 9193 (2011).

37. N. Lindvall, M. T. Cole, and A. Yurgens, Applied Physics Letters, 98 (2011).

38. A. Turchanin, D. Weber, M. Buenfeld, C. Kisielowski, M. V. Fistul, K. B. Efetov, T. Weimann, R. Stosch, J. Mayer, and A. Golzhauser, Acs Nano, 5, 3896 (2011)

39. Z. Zhang, B. Ge, Y. Guo, D. Tang, X. Wang, and F. Wang, Chem Commun (Camb), 49, 2789 (2013).

40. K. H. Lee, H. J. Shin, B. Kumar, H. S. Kim, J. Lee, R. Bhatia, S. H. Kim, I. Y. Lee H. S. Lee, G. H. Kim, J. B. Yoo, J. Y. Choi, and S. W. Kim, Angew Chem Int Ed Engl, 53, 11493 (2014).

41. C. N. Shyam Kumar, V. S. K. Chakravadhanula, A. Riaz, S. Dehm, D. Wang, X. Mu, B. Flavel, R. Krupke, and C. Kubel, Nanoscale, 9, 12835 (2017)

42. P. J. Wessely, F. Wessely, E. Birinci, K. Beckmann, B. Riedinger, and U. Schwalke, Physica E: Low-dimensional Systems and Nanostructures, 44, 1132 (2012).

43. D. Noll and U. Schwalke, in 2016 International Conference on Design and Technology of Integrated Systems in Nanoscale Era (DTIS), p. 1 (2016).

44. A. Boyd, I. Dube, G. Fedorov, M. Paranjape, and P. Barbara, Carbon, 69, 417 (2014)

45. R. K. Srivastava, P. Lal, R. Dwivedi, and S. K. Srivastava, Sensor Actuat B-Chem, 21, 213 (1994).

46. F. Long, P. Yasaei, R. Sanoj, W. Yao, P. Kral, A. Salehi-Khojin, and R. Shahbazian-Yassar, ACS Appl Mater Interfaces, 8, 18360 (2016).

47. H. Wang, Y. Wu, C. Cong, J. Shang, and T. Yu, ACS Nano, 4, 7221 (2010).

48. P. Joshi, H. E. Romero, A. T. Neal, V. K. Toutam, and S. A. Tadigadapa, J Phys Condens Matter, 22, 334214 (2010). 